\title{
Successful Removal from the Duodenum of Swallowed Sewing Needles Using Devised Endoscopic Forceps
}

We report on our novel technique for endoscopic removal of sharp needles from the duodenum. A 43-year-old woman was referred to our hospital after attempting suicide by swallowing sewing needles. Endoscopy revealed the presence of five needles in the duodenum, with the tips lodged in the duodenal wall (Figure 1). Four of the five needles were removed safely using a snare and grasping forceps (SD11L-1, FG-17K-1; Olympus, Tokyo, Japan) through an overtube in the esophagus. The fifth needle had penetrated through the pylorus into the stomach during the procedure. To remove it without further injury, we used forceps (FB$21 \mathrm{~K}-1$; Olympus) through the sheath normally used for a clip device (HX-5LR-1; Olympus). After placing the needle completely within the sheath, we were able to remove the needle through the forceps channel easily and safely (Figure 2 ).

To remove ingested foreign bodies, endoscopic approaches are generally less invasive than surgical intervention. However, extreme caution is required when the foreign body is sharp, as it is easy to injure the wall of the gastrointestinal tract. One method involves using an overtube to protect the esophagus during repeated insertion and withdrawal of the endoscope $[1,2]$. Another technique involves fashioning a protective hood for the endoscope

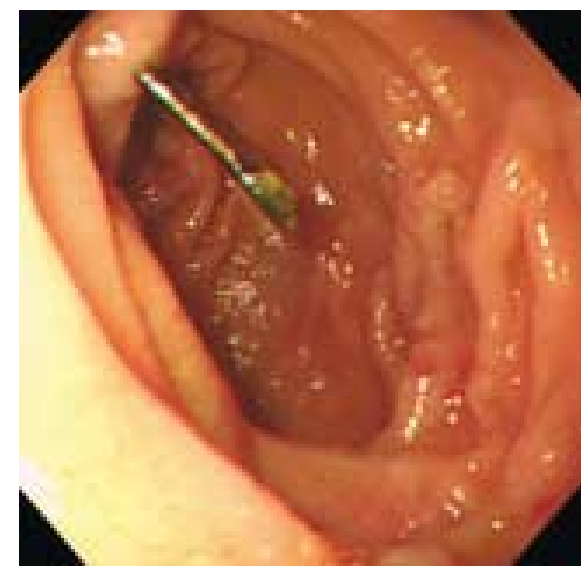

Figure 1 Endoscopic view of the duodenum. The needle had become stuck in the wall of the duodenum. using variety of devices, such as soft latex protector hoods $[3,4]$. Using the endoscopic approach shown here, we were able to remove the needles without complication.

For this procedure, we used grasping forceps through the sheath normally used for a clip device. The fifth needle was placed within the sheath, and was covered completely. Thus, all the needles were taken out through the forceps channel without repeating insertion of the endoscope itself. If the endoscope can reach the site, it is possible to remove sharp foreign bodies from the gastrointestinal safe endoscopic removal of the sharp needles.

\section{A. Enjoji', Y. Nagata' ${ }^{1}$ A. Furuichi', \\ S. Kawakami', H. Saiwai', J. Furui', K. Kawazoe ${ }^{2}$, T. Kanematsu ${ }^{1}$}

${ }^{1}$ Department of Transplantation and Digestive Surgery, Nagasaki University Graduate School of Biomedical Sciences, Nagasaki, Japan

2 Department of Endoscopy, Nagasaki University School of Medicine, Nagasaki, Japan.
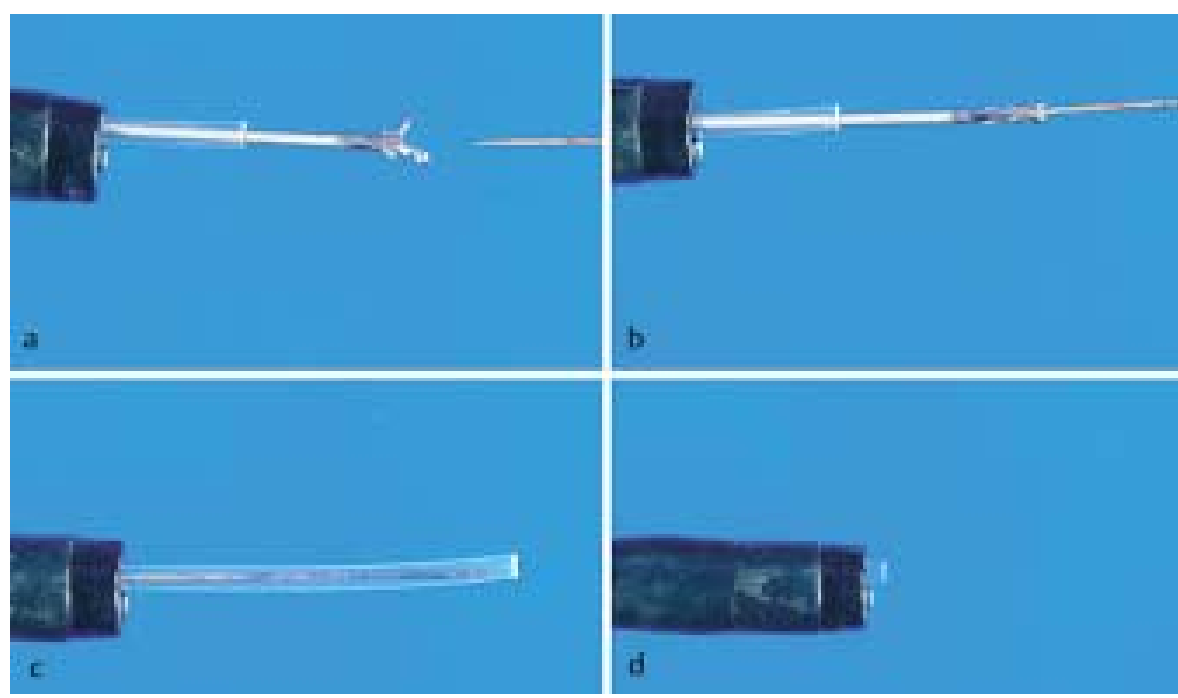

Figure 2 a Forceps were inserted through the sheath of a clip device. $\mathbf{b}$ The head of the neddle was grasped with the forceps. $\mathbf{c}$ The entire needle was drawn into the sheath. $\mathbf{d}$ The sheath and needle were removed through the forceps channel of the endoscope.

${ }^{1}$ Rogers BH, Kot C, Meiri S, Epstein M. An overtube for the flexible fiberoptic esophagogastroduodenoscope. Gastrointest Endosc 1982; 28: 256-257

2 Spurling TJ, Zaloga GP, Richter JE. Fiberendoscopic removal of a gastric foreign body with overtube technique. Gastointest Endosc 1983; 29: 226-227

${ }^{3}$ Bertoni G, Pacchione D, Conigliaro R et al. Endoscopic protector hood for safe removal of sharp-pointed gastroesophageal foreign bodies. Surg Endosc 1992; 6: $255-258$

${ }^{4}$ Bertoni G, Sassatelli R, Conigliaro R et al. A simple latex protector hood for sage endoscopic removal of sharp-pointed gastroesophageal foreign bodies. Gastrointest Endosc 1996; 44: 458-461

\section{Corresponding Author}

\section{A. Enjoji, M.D.}

Department of Transplantation and Digestive Surgery, Nagasaki University Graduate School of Biomedical Sciences 1-7-1 Sakamoto, Nagasaki 852-8501,Japan Fax: $\quad$ +81-958-497319

E-mail: aenjoji@net.nagasaki-u.ac.jp tract. Our technique allowed easy and 